\title{
Targeting ER $\alpha$-glucosidase I with a single-dose iminosugar treatment protects against lethal influenza and dengue virus infections
}

Kelly L. Warfield ${ }^{1 *}$, Dominic S. Alonzi 2 \$, Johan C. Hill ${ }^{2}$, Alessandro T. Caputo ${ }^{2}$, Pietro Roversi 2,6 , J. L. Kiappes ${ }^{2}$, Nicholas Sheets ${ }^{3}$, Matthew Duchars ${ }^{1}$, Raymond A. Dwek ${ }^{2}$, Julia Biggins ${ }^{4}$, Dale Barnard $^{5}$, Sujan Shresta ${ }^{3}$, Anthony M. Treston ${ }^{1 \#}$ and Nicole Zitzmann ${ }^{2 \# *}$.

1:Emergent BioSolutions, Gaithersburg, MD, 20879, United States

2:Oxford Glycobiology Institute, Department of Biochemistry, University of Oxford, South Parks Road, Oxford OX1 3QU, England, United Kingdom;

3:La Jolla Institute for Allergy and Immunology, La Jolla, CA, 92037, USA;

4: Integrated Biotherapeutics Inc., Gaithersburg, MD, 20878, United States

5: Institute for Antiviral Research, Utah State University, Logan, UT, 84322, United States

6: Leicester Institute of Structural and Chemical Biology, Department of Molecular and Cell Biology, University of Leicester, Henry Wellcome Building, Lancaster Road, Leicester, LE1 7RH, England, United Kingdom.

\section{ASSOCIATED CONTENT}

Supporting Information

Supplementary Figure 1. Free Oligosaccharide analysis from UV-4B single dosed mice Supplementary Figure 2. Isothermal titration calorimetry of ER Glu I with iminosugars Supplementary Table 1. Statistical analysis of Figure 1A-B data Supplementary Table 2. Statistical analysis of Figure 1C data Supplementary Table 3. Statistical analysis of Figure 2A data Supplementary Table 4. Data collection and refinement statistics 


\section{Supplementary Figures}

Supplementary Figure 1. Free Oligosaccharide analysis from UV-4B single dosed mice. Time course monitoring levels of $\mathrm{Glc}_{3} \mathrm{Man}_{7} \mathrm{GlcNAc}_{2}$ following a single dose of $750 \mathrm{mg} / \mathrm{kg} / \mathrm{day}$ or $250 \mathrm{mg} / \mathrm{kg} /$ day of UV4, at $1,2,4,8$ and 16 hours post administration in (a) serum and (b) spleen ( $\mathrm{n}=5$ per group).
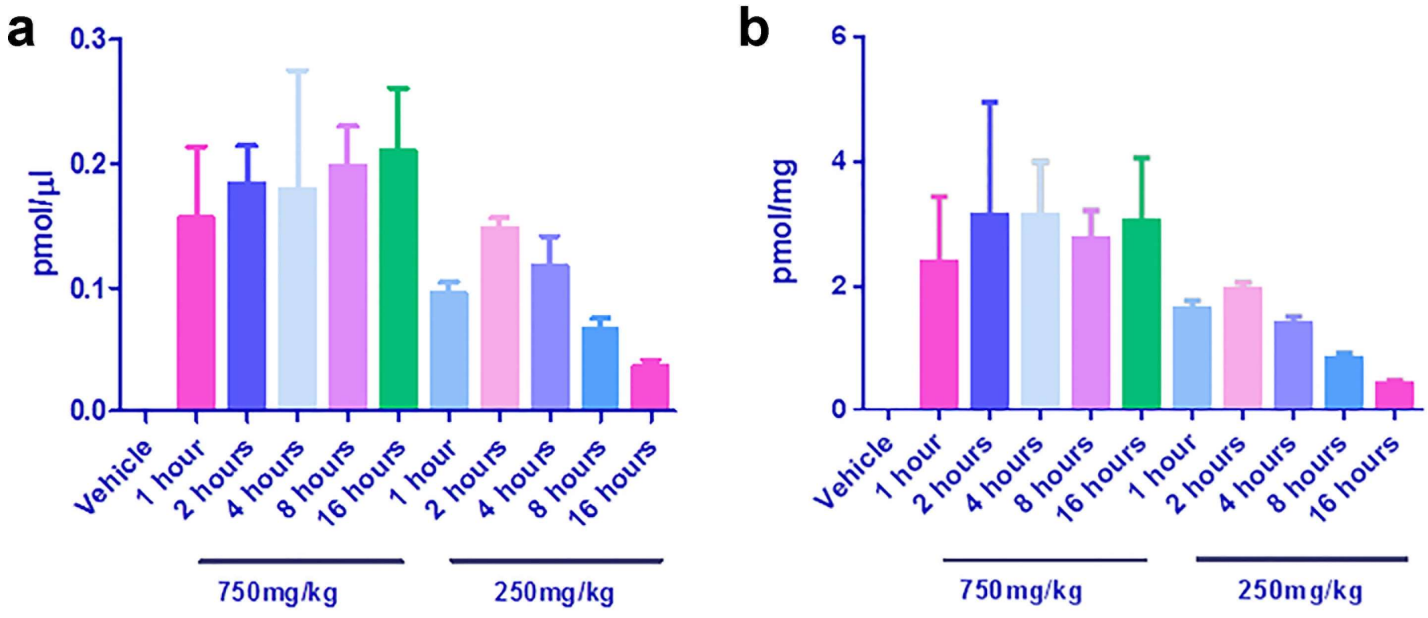

Supplementary Figure 2. Isothermaltitration calorimetry of Glu I with iminosugars. Glu I was titrated alkylated iminosugars. (A) UV-4B, KD of $212 \mathrm{nM}(157-325 \mathrm{nM}), \Delta$ S of $-5.92 \mathrm{cal} / \mathrm{mol} / \mathrm{deg}$ and $\Delta \mathrm{H}$ of $-1.087 \mathrm{E} 4$ $( \pm 346.8) \mathrm{cal} / \mathrm{mol}$. (B) $N$-butyl-DNJ, KD of $463 \mathrm{nM}(352-698 \mathrm{nM}), \Delta$ S of $-15.2 \mathrm{cal} / \mathrm{mol} / \mathrm{deg}$ and $\Delta \mathrm{H}$ of $-1.317 \mathrm{E} 4$ $( \pm 430.3) \mathrm{cal} / \mathrm{mol}$.

A

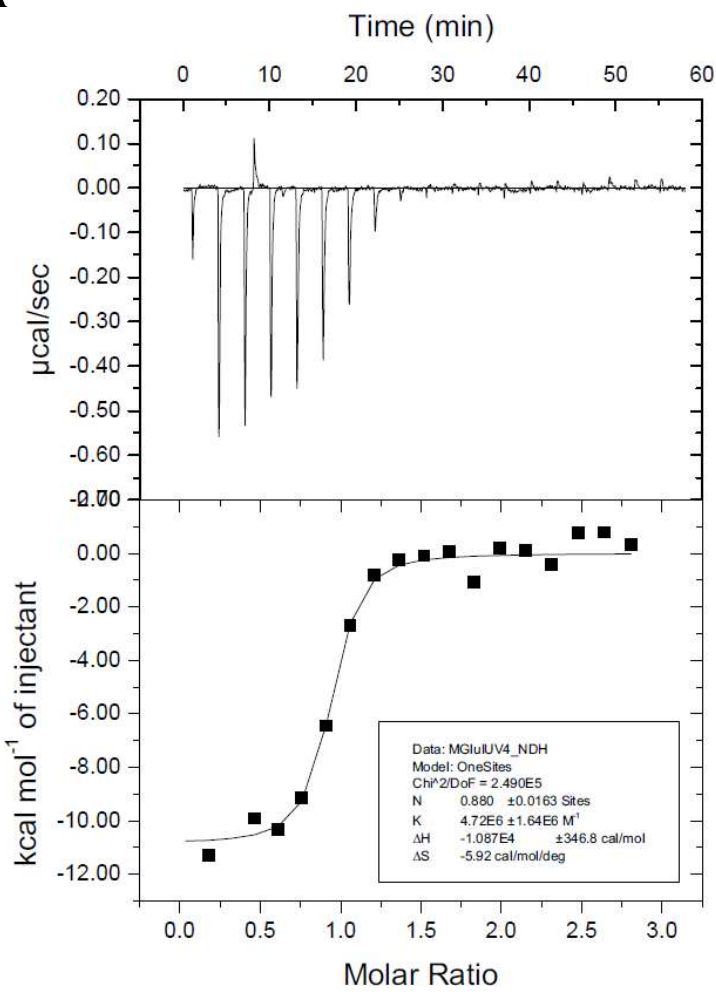

B

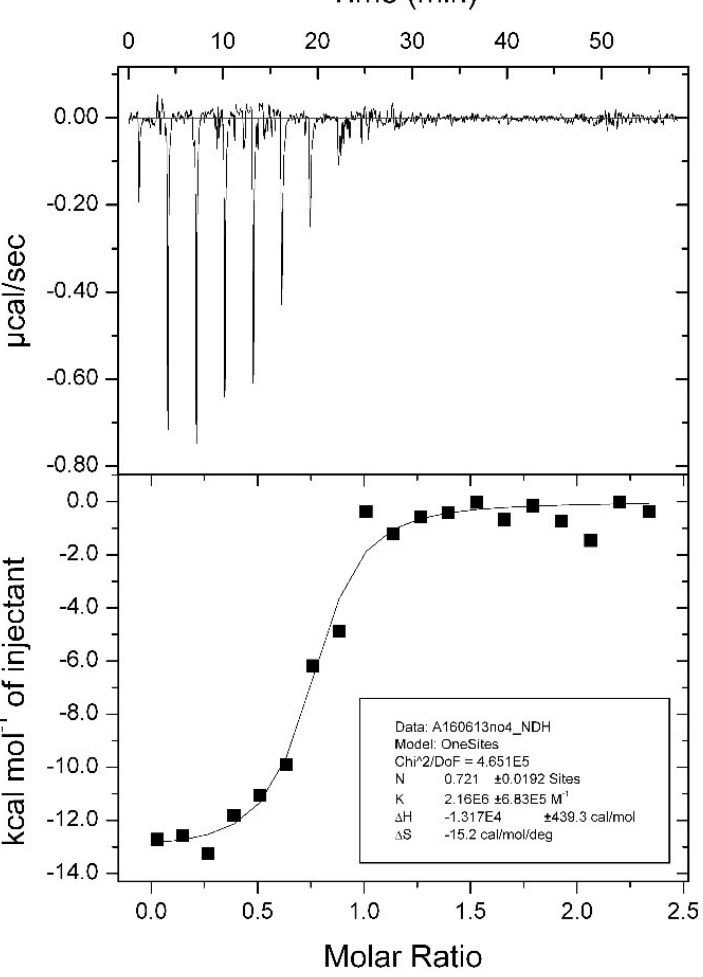




\section{Supplementary Tables}

Supplementary Table 1. Statistical analysis of Figure 1A-B data. Groups of female 6-8 week old $\mathrm{BALB} / \mathrm{c}$ mice were infected intranasally (IN) with a $1 \mathrm{xLD}_{90}$ of mouse-adapted influenza A/Texas/36/91 (H1N1) and then given a single dose of $0,100,250,500,750$, or $1000 \mathrm{mg} / \mathrm{kg}$ of UV-4 approximately 8 hours post infection (hpi). Additional groups of mice were administered multiple doses of either an efficacious $(100 \mathrm{mg} / \mathrm{kg})$ or sub-efficacious $(20 \mathrm{mg} / \mathrm{kg})$ dose of UV-4 or vehicle (water) only starting $8 \mathrm{hpi}$ and continuing thrice daily (TID) for a total of 5 days. The results presented are a combination of results from two independentstudies.

\begin{tabular}{|c|c|c|c|c|c|c|}
\hline Group & $\begin{array}{c}\text { UV-4B } \\
\text { dose } \\
\text { level } \\
(\mathbf{m g} / \mathbf{k g})^{\mathrm{a}}\end{array}$ & Regimen & $\begin{array}{l}\text { Number } \\
\text { of } \\
\text { animals }\end{array}$ & Survival & $\begin{array}{l}\text { Median } \\
\text { survivale }\end{array}$ & $\begin{array}{l}\text { P value compared to } \\
\text { water treatment }\end{array}$ \\
\hline 1 & 1000 & \multirow{6}{*}{ Once at $8 \mathrm{hpi}$} & 20 & $70 \%$ & $>14$ & 0.0002 \\
\hline 2 & 750 & & 20 & $90 \%$ & $>14$ & $<0.0001$ \\
\hline 3 & 500 & & 20 & $60 \%$ & $>14$ & 0.0003 \\
\hline 4 & 250 & & 20 & $30 \%$ & 10 & 0.0778 \\
\hline 5 & 100 & & 20 & $25 \%$ & 10 & 0.0046 \\
\hline 6 & Vehicle & & 20 & $10 \%$ & 10 & N/A \\
\hline 7 & 100 & \multirow{3}{*}{ TID for 5 days } & 20 & $85 \%$ & $>14$ & $<0.0001$ \\
\hline 8 & 20 & & 10 & $20 \%$ & 10 & 0.0852 \\
\hline 9 & Vehicle & & 20 & $20 \%$ & 9 & N/A \\
\hline
\end{tabular}

a Doses are calculated and reported as equivalents of the active component UV-4 free base. All dosing was via oral gavage

b Kaplan-Meier survival curves were analyzed using GraphPad Prism using the Mantel-Cox log-rank test followed by the Gehan-Breslow-Wilcoxon test. $\mathrm{P}<0.05$ indicates statistical significance from indicated comparison group.

${ }^{c}$ If mortality did not exceed $50 \%$ at the end of the study then the median survival could not be computed and is therefore reported as $>14$ days. This is reported as 'undefined' in GraphPad Prism. N/A - Not Applicable 
Supplementary Table 2. Statistical analysis of Figure 1C data. Groups of 10 female 6-8 week old $\mathrm{BALB} / \mathrm{c}$ mice were infected intranasally (IN) with a $1 \mathrm{xLD}_{90}$ of mouse-adapted influenza A/PA/10/2010 (H3N2)sw and then given a single dose of $0,100,250,500,750$, or $1000 \mathrm{mg} / \mathrm{kg}$ of UV-4 approximately 8 hours post infection (hpi). An additional group of mice in this study was given $10 \mathrm{mg} / \mathrm{kg}$ of oseltamivir via oral gavage starting 8 hpi and continuing twice daily (BID) for a total of 6 days.

\begin{tabular}{|c|c|c|c|c|c|c|c|}
\hline Group & Treatment & $\begin{array}{c}\text { UV-4B } \\
\text { Dose level } \\
(\mathrm{mg} / \mathrm{kg})^{\mathrm{a}}\end{array}$ & Regimen & $\begin{array}{l}\text { Number } \\
\text { of animals }\end{array}$ & Survival & $\begin{array}{l}\text { Median } \\
\text { Survival } \\
\text { (Days) }\end{array}$ & $\begin{array}{c}\text { P value } \\
\text { compared to } \\
\text { water } \\
\text { treatment }\end{array}$ \\
\hline $\mathbf{1}$ & UV-4B & 1000 & Once at $8 \mathrm{hpi}$ & 10 & $100 \%$ & $>14$ & $<0.0001$ \\
\hline 2 & & 750 & & 10 & $80 \%$ & $>14$ & 0.0117 \\
\hline 3 & & 500 & & 10 & $40 \%$ & 9 & 0.9669 \\
\hline 4 & & 250 & & 9 & $78 \%$ & $>14$ & 0.0439 \\
\hline 5 & & 100 & & 10 & $30 \%$ & 10 & 1.0000 \\
\hline 6 & Vehicle & Vehicle & & 10 & $33 \%$ & 9 & $\mathrm{~N} / \mathrm{A}$ \\
\hline 7 & Oseltamivir & 10 & BID for 6 days & 10 & $100 \%$ & $>14$ & $<0.0001$ \\
\hline
\end{tabular}

a Doses are calculated and reported as equivalents of the active component UV-4 free base. All dosing was via oral gavage

${ }^{\mathrm{b}}$ Kaplan-Meier survival curves were analyzed using GraphPad Prism using the Mantel-Cox log-rank test followed by the Gehan-Breslow-Wilcoxon test. $\mathrm{P}<0.05$ indicates statistical significance from indicated comparison group.

${ }^{c}$ If mortality did not exceed $50 \%$ at the end of the study then the median survival could not be computed and is therefore reported as $>14$ days. This is reported as 'undefined' in GraphPad Prism.

N/A - Not Applicable 
Supplementary Table 3. Statistical analysis of Figure 2A data. AG129 mice of both sexes were split evenly into treatment groups ( $\mathrm{n}=7-8 /$ group). The mice received $5 \mu \mathrm{g}$ of $2 \mathrm{H} 2$ (anti-prM/M) antibody one hour before infection with $1 \mathrm{XLD}_{90}$ dose of DENV2-S221 intravenously. Groups of mice received 0,250 , $375,500,750$, or $1000 \mathrm{mg} / \mathrm{kg}$ of UV-4 once approximately $8 \mathrm{hpi}$. The predefined study endpoints were mortality, euthanasia due to severe morbidity, or termination after 10 days post viral infection. The results presented are a combination of results from two independent studies.

\begin{tabular}{|c|c|c|c|c|c|c|}
\hline Group & $\begin{array}{c}\text { UV-4B } \\
\text { dose } \\
\text { level } \\
(\mathbf{m g} / \mathbf{k g})^{a}\end{array}$ & Regimen & $\begin{array}{c}\text { Number } \\
\text { of } \\
\text { animals }\end{array}$ & Survival & $\begin{array}{l}\text { Median } \\
\text { Survival } \\
\text { (Days)c }^{c}\end{array}$ & $\begin{array}{l}\text { P value compared to } \\
\text { water treatment }\end{array}$ \\
\hline 1 & 1000 & \multirow[t]{6}{*}{ Once at $8 \mathrm{hpi}$} & 8 & $70 \%$ & $>10$ & 0.0002 \\
\hline 2 & 750 & & 15 & $90 \%$ & $>10$ & 0.0005 \\
\hline 3 & 500 & & 8 & $60 \%$ & $>10$ & 0.0014 \\
\hline 4 & 375 & & 7 & $30 \%$ & 8 & 0.0138 \\
\hline 5 & 250 & & 15 & $25 \%$ & 6 & 0.0081 \\
\hline 6 & Vehicle & & 16 & $10 \%$ & 5 & $\mathrm{~N} / \mathrm{A}$ \\
\hline 7 & 100 & TID for 5 days & 8 & $85 \%$ & $>10$ & 0.0002 \\
\hline
\end{tabular}

${ }^{a}$ Doses are calculated and reported as equivalents of the active component UV-4 free base. All dosing was via oral gavage

${ }^{\mathrm{b}}$ Kaplan-Meier survival curves were analyzed using GraphPad Prism using the Mantel-Cox log-rank test followed by the Gehan-Breslow-Wilcoxon test. $\mathrm{P}<0.05$ indicates statistical significance from indicated comparison group.

c If mortality did not exceed $50 \%$ at the end of the study then the median survival could not be computed and is therefore reported as $>10$ days. This is reported as 'undefined' in GraphPad Prism.

N/A - Not Applicable 


\begin{tabular}{|c|c|}
\hline & $\begin{array}{l}\text { MmGluI with UV-4B } \\
\text { (5MHF) }\end{array}$ \\
\hline \multicolumn{2}{|l|}{ Data collection } \\
\hline $\begin{array}{l}\text { Space group } \\
\text { Cell dimensions }\end{array}$ & P $12{ }_{1} 1$ \\
\hline $\begin{array}{l}\quad a, b, c(\AA) \\
\quad a, b, g\left(^{\circ}\right) \\
\text { Resolution }(\AA) \\
R_{\text {merge }}, R_{\text {meas }} \text {, and } R_{\text {pim }} \\
I / \sigma(I) \\
C C_{1 / 2} \\
\text { Completeness }(\%) \\
\text { Redundancy }\end{array}$ & $\begin{array}{c}108.01130 .92135 .37 \\
90.00099 .5399 .000 \\
133.5-2.1(2.11-2.10)^{\mathrm{a}} \\
0.105(0.607), 0.125(0.715) \\
0.067(0.376) \\
8.1(2.1) \\
0.997(0.853) \\
99.5(96.8) \\
3.4(3.5)\end{array}$ \\
\hline \multicolumn{2}{|l|}{ Refinement } \\
\hline $\begin{array}{l}\text { Resolution }(\AA) \\
\text { No. reflections } \\
R_{\text {work }} / R_{\text {free }} \\
\text { No. atoms }\end{array}$ & $\begin{array}{c}2.38^{\mathrm{b}} \\
214780(15781)^{\mathrm{b}} \\
0.188 / 0.216\end{array}$ \\
\hline $\begin{array}{l}\text { Protein } \\
\text { Ligand/ion } \\
\text { (specify/describe) }\end{array}$ & $\begin{array}{c}24042 \\
4 x\left(6 \mathrm{~A} 9,3 \times \mathrm{SO}_{4}, 1 \times 7 \mathrm{PG}\right)^{\mathrm{c}}\end{array}$ \\
\hline $\begin{array}{l}\text { Water } \\
B \text { factors }\left(\mathrm{Ai}^{2}\right)\end{array}$ & 1387 \\
\hline $\begin{array}{l}\text { Protein } \\
\text { Ligand/ion } \\
\text { Water }\end{array}$ & $\begin{array}{l}\text { A: } 41.7, \text { B: } 45.1, \text { C: } 44.4, \text { D: } 43.2 \\
\text { A: } 46.6, \text { B: } 52.5, \text { C: } 50.6, \text { D: } 46.0 \\
\text { A: } 41.0, \text { B: } 47.0, \text { C: } 45.7, \text { D: } 44.9\end{array}$ \\
\hline R.m.s. deviations & \\
\hline $\begin{array}{l}\text { Bond lengths }(\AA) \\
\text { Bond angles }\left({ }^{\circ}\right)\end{array}$ & $\begin{array}{c}0.010 \\
1.08\end{array}$ \\
\hline
\end{tabular}

a Values in parentheses are for highest-resolution shell.

b Data were scaled to $2.10 \AA$ using anisotropic data from the Autoproc programme, StarAniso.

c $6 \mathrm{~A} 9$ is known as UV4, 7PG as PEG 